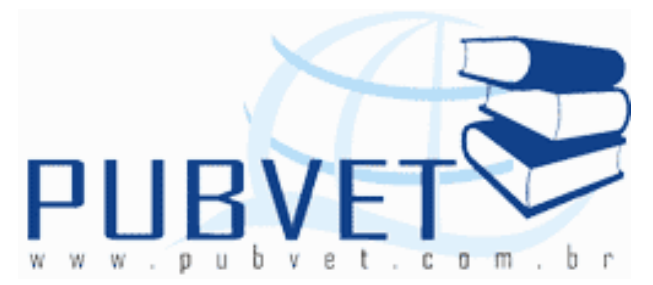

PUBVET, Publicações em Medicina Veterinária e Zootecnia.

\title{
Importância das vacinas sintéticas contra o carrapato Rhipicephalus microplus
}

\author{
Gabriel Domingos Carvalho ${ }^{1}$, Ana Paula Peconick ${ }^{2}$, Marlene Isabel Vargas ${ }^{3}$, \\ Joaquín Hernán Patarroyo ${ }^{4}$
}

${ }^{1}$ Professor do Instituto Federal Norte de Minas Gerais - IFNMG Campus Salinas, Salinas, MG, Brasil. E-mail: gabriel.carvalho@ifnmg.edu.br

${ }^{2}$ Professora Adjunta - Departamento de Medicina Veterinária - Universidade Federal de Lavras (UFLA), Lavras, MG, Brasil.

${ }^{3}$ Professora Adjunta - Departamento de Veterinária - Universidade Federal de Viçosa (UFV), Viçosa, MG, Brasil.

${ }^{4}$ Professor Titular - Departamento de Veterinária - Universidade Federal de Viçosa (UFV), Viçosa, MG, Brasil.

\section{Resumo}

Rhipicephalus microplus, do ponto de vista econômico, é o parasita mais importante dos rebanhos bovinos nas Américas Central e Sul e na Austrália, pois acarreta severos prejuízos diretos e indiretos na produção animal. $O$ controle deste parasita repousa principalmente na utilização de drogas carrapaticidas que são responsáveis pela seleção de populações resistentes, além de causar a contaminação com resíduos na carne, leite e derivados e no meio ambiente. Isso gera uma necessidade de se desenvolver métodos alternativos, seguros e eficientes de controle, os quais possam atuar de modo 
integrado. Os avanços das técnicas de biologia molecular impulsionaram várias linhas de pesquisa na área de parasitologia veterinária, principalmente no desenvolvimento de vacinas. Vacinas derivadas da glicoproteína intestinal do R. microp/us, a Bm86, possuem um amplo potencial como método não químico no controle dos carrapatos. O peptídeo sintético SBm7462 ${ }^{\circledR}$, uma vacina sintética derivada da Bm86, a qual tem demonstrado sua eficácia na indução da resposta imune dos animais com ela imunizados.

Palavras-chave: vacinas sintéticas, carrapato, Rhipicephalus (Boophilus) microplus.

\section{Importance of synthetic vaccines ant-tick Rhipicephalus microplus}

\section{Abstract}

Rhipicephalus microplus, from an economic standpoint, is the most important parasite of cattle herds in Central and South America and Australia, it causes severe direct and indirect losses in animal production. Control of this parasite rests primarily on the use of acaricides drugs that are responsible for the selection of resistant of populations and cause contamination with residues in meat, dairy products and in the environment. This creates a need to the development of alternative, safe and efficient control, which may act in an integrated manner. Advances in molecular biology techniques have driven several lines of research in veterinary parasitology, particularly in vaccine development. Vaccines derived from the intestinal glycoprotein of $R$. microp/us, the Bm86, have broad potential as a non-chemical control of ticks. The synthetic peptide $\mathrm{SBm} 7462^{\circledR}$, a synthetic vaccine derivative of the $\mathrm{Bm} 86$, has demonstrated its effectiveness in inducing the immune response of animals immunized with it.

Keywords: synthetic vaccine, tick, Rhipicephalus (Boophilus) microplus. 
CARVALHO, G.D. et al. Importância das vacinas sintéticas contra o carrapato Rhipicephalus microplus. PUBVET, Londrina, V. 8, N. 10, Ed. 259, Art. 1716, Maio, 2014.

\section{INTRODUÇÃO}

O carrapato Rhipicephalus microplus representa o principal ectoparasito de bovinos nas regiões tropicais e subtropicais, e o seu controle repousa principalmente na utilização de fármacos carrapaticidas, os quais propiciam a seleção de populações resistentes (SANGSTER, 2001; TAYLOR, 2001). A dependência exclusiva de compostos químicos para o controle desse parasita tem-se tornado uma das principais preocupações técnico-científicas e econômicas da atualidade, daí a necessidade de se desenvolver vacinas capazes de induzir a imunidade do hospedeiro contra o parasita.

A demanda por vacinas parasitárias em saúde animal é crescente, e empresas remetem lucros acima de três bilhões de dólares somente nesse setor (DALTON; MULCAHY, 2001). O avanço das técnicas de biologia molecular providencia ferramentas para compreender muitas questões biológicas, sendo esses métodos aplicados em várias linhas de pesquisa na área de parasitologia veterinária (SANGSTER et al., 2001). O sucesso na prevenção e controle de doenças parasitárias advém do melhor conhecimento das interações hospedeiro-parasita e também de estudos nas áreas da genômica, proteômica e bioinformática, além dos avanços nas áreas da engenharia genética (PRICHARD; TAIT, 2001).

O Laboratório de Biologia e Controle de Hematozoários e Vetores (LBCHV/BIOAGRO/DVT/UFV) vem desenvolvendo pesquisas visando o constante aprimoramento da vacina sintética anti-carrapato $R$. microplus $\left(\mathrm{SBm} 7462{ }^{\circledR}\right)$, de comprovada eficiência, a qual já foi patenteada no Brasil, USA, México, Austrália e União Européia. Os imunógenos sintéticos foram desenhados para estimular uma resposta imune apropriada, incluindo epítopos de células $B$ e $T$ relevantes e excluindo aquelas regiões da proteína que poderiam atuar estimulando a atividade de mecanismos supressores, alérgicos e/ou autoimunes. PATARROYO et al. (2002) demonstraram a eficácia de vacinas sintéticas na indução de uma forte atividade estimuladora das células linfóides por meio dos peptídeos sintéticos. 
CARVALHO, G.D. et al. Importância das vacinas sintéticas contra o carrapato Rhipicephalus microplus. PUBVET, Londrina, V. 8, N. 10, Ed. 259, Art. 1716, Maio, 2014.

\section{REVISÃO DE LITERATURA}

\section{Importância do carrapato Rhipicephalus microplus}

Dentre as 869 espécies conhecidas de carrapatos no mundo, o Rhipicephalus microplus (CANESTRINI, 1887) representa o principal ectoparasito de bovinos nas regiões de clima tropical e subtropical. Estudos recentes utilizando metodologias taxonômicas moleculares, demonstraram a proximidade filogenética do gênero Boophilus com o Rhipicephalus, propondose então uma nova classificação para a espécie, que mudaria de Boophilus microplus para Rhipicephalus (Boophilus) microplus (MURREL et al., 2000; MURREL et al., 2001; BEATI; KEIRANS, 2001). A classificação definida por NUÑEZ et al. (1982) com as atualizações propostas seguiria a seguinte ordem:

Filo: Arthropoda

Classe: Arachnida

Ordem: Acari

Subordem: Metastigmata

Família: Ixodidae

Subfamília: Rhipicephalinae

Gênero: Riphicephalus

Espécie: Rhipicephalus (Boophilus) microplus

Seguindo a mesma linha de pesquisa taxonômica, estudos apontam para uma origem africana da subfamília Rhipicephalinae (MURREL et al., 2000; MURREL et al., 2001; BARKER e MURRELL, 2002). Ao longo dos séculos, o $R$. microplus se difundiu pelas regiões neo-tropicais juntamente com as migrações de pessoas e suas criações de animais. Importantes divergências genéticas e adaptativas da espécie, distribuída entre os diferentes continentes, envolvem uma "plasticidade ecológica" das populações de carrapatos, onde as mudanças globais afetam os parasitas e a forma manejo e controle dos mesmos (SUTHERST, 2001; ESTRADA-PEÑA et al., 2006). Na América Latina, o $R$. microplus encontra-se amplamente distribuído pelo Brasil, norte da Argentina, 
CARVALHO, G.D. et al. Importância das vacinas sintéticas contra o carrapato Rhipicephalus microplus. PUBVET, Londrina, V. 8, N. 10, Ed. 259, Art. 1716, Maio, 2014.

Paraguai, Uruguai, leste da Bolívia, Colômbia e Venezuela. No Brasil, destacam-se as regiões Centro-Oeste e Sudeste, locais com intensa atividade pecuária e que, além do hospedeiro, possuem condições ideais de temperatura e umidade para o desenvolvimento do parasita (ESTRADA-PEÑA, 1999; ESTRADA-PEÑA et al., 2006).

O carrapato $R$. microplus acarreta severos prejuízos diretos e indiretos na produção animal. Os primeiros estão relacionados com a perda de peso, baixa conversão alimentar, perdas na qualidade do couro, toxinas liberadas no hospedeiro, lesões de pele, entre outros. JONSSON (2006) calculou que cada fêmea ingurgitada de $R$. microplus é responsável por uma redução de aproximadamente $1,37 \mathrm{~g}$ de peso dos bovinos, além causar anemia, supressão do apetite e alteração no metabolismo dos animais infestados. As perdas indiretas estão relacionadas com a transmissão de hematozoários, como as espécies dos gêneros Babesia e Anaplasma (PATARROYO, 1994; SANTOS et al., 1998; RUIZ et al., 2005; OLIVEIRA-SIQUEIRA et al., 2005). Estudos demonstram que os carrapatos provocam efeitos imunossupressores no hospedeiro, o que pode facilitar a transmissão ou a gravidade nos casos babesioses e anaplasmoses (KASHINO et al., 2005; JONSSON, 2006).

Os prejuízos causados pela infestação de carrapatos e as doenças transmitidas por eles, segundo a FAO (Food and Agriculture Organization), ultrapassam sete bilhões de dólares anuais no mundo. Somente no Brasil, estudos realizados por GRISI et al. (2002) apontaram prejuízos superiores a dois bilhões de dólares anuais.

O controle de carrapatos repousa principalmente na utilização de drogas carrapaticidas que são responsáveis pela seleção de populações resistentes (SANGSTER, 2001; TAYLOR, 2001). Outro problema com o uso de acaricidas é a contaminação com resíduos na carne, leite e derivados e no meio ambiente. Tudo isso aponta para a necessidade de pesquisas de métodos alternativos, seguros e eficientes de controle, os quais possam atuar de modo integrado (WILLADSEN, 2006). 
CARVALHO, G.D. et al. Importância das vacinas sintéticas contra o carrapato Rhipicephalus microplus. PUBVET, Londrina, V. 8, N. 10, Ed. 259, Art. 1716, Maio, 2014.

\section{Vacinas contra Rhipicephalus microplus}

O processo de vacinação é o método que apresenta a melhor relação custo-benefício para prevenir perdas econômicas e aumentar a qualidade de vida dos animais domésticos (AUCOUTURIER et al., 2001). No âmbito da Medicina Veterinária, muitos imunógenos ainda são produzidos utilizando-se tecnologia convencional, tais como vacinas atenuadas. Porém, com o desenvolvimento de pesquisas que aplicam ferramentas de biotecnologia, estas, estão sendo usadas no desenvolvimento de vacinas. Essas vacinas "modernas" não são utilizadas apenas para o controle de doenças infecciosas, mas também para aumentar a produtividade do rebanho e o controle de ectoparasitos (BABIUK, 2002).

O processo de vacinação representa uma medida preventiva que oferece vantagens sobre os métodos convencionais de controle químicos. Segundo WILLADSEN (2004) este processo representa uma ação sustentável, livre de resíduos, mais específica e com menores problemas de resistência quando comparado com os fármacos utilizados comumente, além da segurança oferecida.

Pesquisas e avanços científicos na área de imunologia favorecem a compreensão cada vez maior sobre a biologia de parasitas. O uso de modernas ferramentas como a biologia molecular e a produção em alta escala permitem que as vacinas parasitárias sejam uma realidade. A vacinação contra carrapatos apresenta maior sucesso do que contra qualquer outro ectoparasita, provavelmente porque eles se alimentam de forma mais lenta do que outros insetos, ficando mais tempo em contato com o sistema imune do hospedeiro, ou talvez pela forma de digestão que eles utilizam (DALTON ; MULCAHY, 2001).

Há mais de 70 anos utiliza-se uma variedade de materiais antigênicos como forma de vacinação contra carrapato, incluindo macerados de todo o carrapato, extratos das glândulas salivares, material intestinal, cutículas, entre outros (WILLADSEN, 2004). Porém, os melhores resultados no processo de vacinação contra carrapatos ocorrem normalmente quando se usa antígenos que não estão expostos durante o processo de alimentação do parasita, sendo 
CARVALHO, G.D. et al. Importância das vacinas sintéticas contra o carrapato Rhipicephalus microplus. PUBVET, Londrina, V. 8, N. 10, Ed. 259, Art. 1716, Maio, 2014.

esses denominados antígenos ocultos ou concealed antigens (OLIVEIRA, 1998).

WILLADSEN et al. (1989) isolaram uma glicoproteína, de 89.000Da de peso molecular e ponto isoelétrico entre 5,1 a 5,6, da membrana intestinal de uma amostra de $R$. (B.) microplus australiana (Yeerongpilly) e denominaram essa proteína de Bm86. Vacinas contendo a Bm86 atuam através de anticorpos anti-Bm86, com o possível envolvimento do complemento e outros mecanismos efetores (DE LA FUENTE et al., 1998; GARCÍA-GARCÍA et al., 1998). O efeito da vacinação é a lise das células intestinais, resultando na redução do número de carrapatos que conseguem completar o seu ciclo de vida, além da menor fertilidade dos carrapatos sobreviventes (RODRIGUEZ et al., 1994).

\section{Vacinas sintéticas contra Rhipicephalus microplus}

A vacina sintética contra o $R$. microplus denominada de $\mathrm{SBm} 7462^{\circledR}$ foi desenvolvida a partir de estudos por predição computacional, definindo-se as possíveis seqüências que continham determinantes imunogênicos. 0 imunógeno possui seqüências que foram desenhadas no Laboratório de Biologia e Controle de Hematozoários e Vetores (LBCHV/BIOAGRO/DVT/UFV) e sintetizadas no Instituto de Inmunologia del Hospital San Juan de Dios, em Bogotá, Colômbia (PATARROYO et al., 2002).

Os imunógenos sintéticos foram desenhados para estimular uma resposta imune apropriada, incluindo-se epítopos B e T relevantes e excluindose aquelas regiões da proteína que poderiam atuar estimulando a atividade de mecanismos supressores, alérgicos e/ou autoimunes. A eficácia da vacina sintética SBm7462 ${ }^{\circledR}$ já foi demonstrada pela indução de uma forte atividade estimuladora das células linfóides por meio de peptídeos sintéticos (PATARROYO et al, 1994). O uso de peptídeos sintéticos como imunógenos tem tido bons resultados, indicando o sucesso no desenvolvimento de vacinas sintéticas (MILICH, 1989).

Estudos de variabilidade genética em $R$. microplus de distintos locais de condições geográficas diferentes, de regiões brasileiras e de outros países sul- 
CARVALHO, G.D. et al. Importância das vacinas sintéticas contra o carrapato Rhipicephalus microplus. PUBVET, Londrina, V. 8, N. 10, Ed. 259, Art. 1716, Maio, 2014.

americanos, demonstraram que a seqüência da vacina sintética $\mathrm{SBm} 7462^{\circledR}$ manteve-se conservada entre todas as populações (SOSSAI et al., 2005; PECONICK et al., 2008), concluindo-se que não há variabilidade nessas seqüências, de modo que pudesse interferir na eficiência vacinal, podendo-se considerá-lo um imunógeno universal, dessa forma, o uso de vacinas sintéticas baseadas no gene bm86 (SBm7462®) serem usadas em diferentes espécies de carrapatos (PECONICK et al., 2008).

Estudos preliminares já haviam demonstrado a eficácia da vacina SBm7462 ${ }^{\circledR}$, por meio de imunizações por via subcutânea, utilizando-se diferentes adjuvantes (PORTELA, 2000; PIMENTEL, 2002; SALES-JUNIOR, 2003, SANCHEZ, 2004). CARVALHO (2008) demonstrou que a vacinação via mucosa, um método não invasivo e indutor de resposta imune sistêmica e local, pode ser considerado viável, sendo as vias mucosas oral e nasal, alternativas para a imunização com o peptídeo sintético SBm7462 ${ }^{\circledR}$. BENEVIDES et al. (2009) utilizando bovinos imunizados com SBm7462 demonstrou que estes apresentaram uma redução no número de fêmeas ingurgitadas, as quais tiveram um decréscimo em sua fertilidade, constatando assim a eficácia desse imunógeno.

\section{CONSIDERAÇÕES FINAIS}

A vacinação representa o melhor método avaliado com custo efetivo para prevenir perdas econômicas e aumentar a duração e qualidade de vida dos animais de produção. Diferentes vacinas são produzidas a partir da proteína intestinal Bm86 do Rhipicephalus microplus. O conhecimento da conservação do gene bm86 é muito importante para avaliar a eficiência de vacinas e a possibilidade de reação cruzada entre espécies diferentes de carrapatos.

Estudos futuros buscarão demonstrar a eficiência das vacinas sintéticas em testes nos bovinos a campo, uma vez que os estudos moleculares demonstraram a importância desse imunógeno.

\section{REFERÊNCIAS}

AUCOUTURIER, J.; DUPUIS, L.; GANNE, V. Adjuvants designed for veterinary and human vaccines. Vaccine, v. 19, p. 2666-2672, 2001. 
BABIUK, L. A. Vaccination: A Management Tool in Veterinary Medicine. The Veterinary Journal, v. 164, p.188-201, 2002.

BARKER, S. C.; MURRELL, A. Phylogeny, evolution and historical zoogeography of ticks: a review of recent progress. Experimental and Applied Acarology, v. 28, p. 55-68, 2002.

BEATI, L.; KEIRANS, J. E. Analysis of the systematic relationships among ticks genera Rhipicephalus and Boophilus (Acari: Ixodidae) based on mitochondrial 12S ribosomal DNA gene sequences and morphological characters. Journal of Parasitology, v. 87, n. 1, p. 3248, 2001.

BENEVIDES, J. A.; ROCHA, H. G. R.; GAMA, M. N.; CAMPOS, V. E. B.; FERREIRA, A. P. B. R.; CARVALHO, G. D.; VALENTE, F. L.; PANIAGO, J. G.; PATARROYO, J. H. Biological parameters of ticks collected from animals immunized with peptides SBbo23290 and SBm7462. Abstracts / Veterinary Immunology and Immunopathology, v. 128, p. 211-347, 2009.

CARVALHO, G.D.; PECONICK, A.P.; MEDEIROS, C.L.; VARGAS-V., M.I.; PATARROYO, J.H. Imunização com o peptídeo sintético anti carrapato Rhipicephalus (Boophilus) microplus SBm7462 por vias de mucosa. Revista Brasileira de Parasitologia Veterinária, v. 17, supl. 1, p. 9-13, 2008.

DALTON, J. P.; MULCAHY, G. Parasite vaccine - a reality? Veterinary Parasitology, v. 98, p. 149-167, 2001.

DE LA FUENTE, J.; RODRÍGUEZ, M.; REDONDO, M.; MONTERO, C.; GARCÍA-GARCÍA, J. C.; MÉNDEZ, L.. SERRANO, E.; VALDÉS, M.; HENRÍQUEZ, A.; CANALES, M.; RAMOS, E.; BOUÉ, O.; MACHADO, H.; LLEONART, R.; DE ARMAS, C. A.; REY, S.; RODRÍGUEZ, J. L.; ARTILES, M.; GARCÍA, L. Field studies and cost-effectiveness analysis of vaccination with Gavac TM against the cattle tick Boophilus microplus. Vaccine, v. 16, p. 336-373, 1998.

ESTRADA-PEÑA, A. Geostatistic and remote sensing using NOAA-AVHRR satellite imagery as predictive tools in tick distribuition and habitat suitability estimations for $R$. (B.) microplus (Acari: Ixodidae) in South America. Veterinary Parasitology, v. 81, p. 73-82, 1999.

ESTRADA-PEÑA, A.; BOUATTOUR, A.; CAMICAS, J. L.; GUGLIELMONE, A.; HORAK, I.; JONGEJAN, F.; LATIF, A.; PEGRAM, R.; WALKER, A. R. The known distribution and ecological preferences of the tick subgenus Boophilus (Acari: Ixodidae) in Africa and Latin America. Experimental and Applied Acarology, v. 38, p. 219-235, 2006.

GARCIA-GARCIA, J. C.; SOTOT, A.; NIGRO, F.; MAZZA, M.; JOGLAR, M.; HECHEVARRIA, M.; LAMBERTI, J.; DE LA FUENTE, J. Adjuvant and immunostimulating properties of the recombinant Bm86 protein expressed in Pichia pastors. Vaccine, v. 16, p. 1053-1055, 1998.

GRISI, L.; MASSARD, C. L.; MOYA-BORJA, G. E.; PEREIRA, J. B. Impacto econômico das principais ectoparasitoses em bovinos no Brasil. A Hora Veterinária, v. 21, n. 125, p. 8-10, 2002.

JONSSON, N. N. The productivity effects of cattle tick (Boophilus microplus) infestation on cattle, with particular reference to Bos indicus cattle and their crosses. Veterinary Parasitology, v. 137, p. 1-10, 2006.

KASHINO, S. S.; RESENDE, J.; SACCO, A. M. S.; ROCHA, C.; PROENÇA, L.; CARVALHO, W. A. ; FIRMINO, A. A.; QUEIROZ, R.; BENAVIDES, M.; GERSHWIN, L. J.; DE MIRANDA-SANTOS, I. K. F. Boophilus microplus: The pattern of bovine immunoglobulin isotype responses to high and low tick infestations. Experimental Parasitology, v. 110, p. 12-21, 2005.

MILICH, D. R. Synthetic $T$ and B cell recognition sites: implications for vaccine development. Asthma and Immunology, v. 45, p. 195-264, 1989.

MURREL, A.; CAMPBELL, N. J. H.; BARKER, S. C. Phylogenetic analyses of Rhipicephaline ticks indicate that the genus Rhipicephalus is paraphiletic. Molecular Phylogenetics and Evolution, v. 16, n. 1, p. 1-7, 2000.

MURRELL, A.; CAMPBELL, N. J. H.; BARKER, S. C. A total-evidence phylogeny of ticks provides insights into the evolution of life cycles and biogeography. Molecular Phylogenetics and Evolution, v. 21, n. 2, p. 244-258, 2001. 
NUÑEZ. J. L.; MUÑOZ, C. M. E.; MOLTEDO, H. L. Boophilus microplus: La Garrapata Común del Ganado Vacuno. Buenos Aires: Hemisferio Sur, 184p. 1982.

OliveirA-SIQUEIRA, T. C. G.; OliVeIRA, M. C. S.; ARAUJO Jr., J. P.; AMARANTE, A. F. T. PCR- based detection of Babesia bovis and Babesia bigemina in their natural host Boophilus microplus and cattle. International Journal for Parasitology, v. 35, p. 105-111, 2005.

OLIVEIRA, R. C. Avaliação experimental do peptídeo sintético 4912 como imunógeno para o controle de carrapato R. (B.) microplus (Canestrini, 1887). 1998. 72 p. Dissertação (Mestrado em Medicina Veterinária). Universidade Federal de Viçosa, Viçosa.

PATARROYO, J. H. Babesiose bovina: controle de vetores com vacinas a base de peptídeos sintéticos. Revista de Patologia Tropical, v. 23, p. 145-146, 1994.

PATARROYO, J.H.; PORTELA, R.W.; DE CASTRO, R.O.; COUTO PIMENTEL, J.; GUZMAN, F.; PATARROYO, M.E.; VARGAS, M.I.; PRATES, A.A.; DIAS MENDES, M.A. Immunization of cattle with synthetic peptides derived from the Boophilus microplus gut protein (Bm86). Veterinary immunology and immunopathology, v. 88, p. 163-172, 2002.

PECONICK, A. P.; SOSSAI, S.; GIRÃO, F. A.; RODRIGUES, M. Q. R.; SOUZA E SILVA, C. H.; GUZMAN, F.; PATARROYO, A. M.; VARGAS, M. I.; PATARROYO, J. H. Synthetic vaccine $\left(\mathrm{SBm} 7462^{\circledR}\right.$ ) against the cattle tick Rhipicephalus (Boophilus) microplus: Preservation of immunogenic determinants in diferent strains from South America. Experimental Parasitology, v. 119, p. 37-43, 2008.

PIMENTEL, J. C. A vacina sintética SBm7462 no controle do carrapato Boophilus microplus (Canestrini, 1887) em animais estabulados e a campo. 2002. 78 p. Dissertação (Mestrado em Medicina Veterinária). Universidade Federal de Viçosa, Viçosa.

PORTELA, R. W. D. Comparação experimental de três peptídeos sintéticos como imunógeno no controle do carrapato Boophilus microplus (Canestrini, 1887). 2000. 87 p. Dissertação (Mestrado em Medicina Veterinária). Universidade Federal de Viçosa, Viçosa.

PRICHARD, R.; TAIT, A. The role molecular biology in veterinary parasitology. Veterinary Parasitology, v. 98, p. 169-194, 2001.

RODRÍGUEZ, M.; RUBIERA, R.; PENICHET, M.; MONTESINO, R.; CREMATA, J.; FALCÓN, V.; SÁNCHEZ, G.; BRINGAS, R.; CORDOBÉS, C.; VALDÉS, M.; LEONART, R.; HERRERA, L.; DE LA FUENTE, J. High-level expresión of the Boophilus microplus BM86 antigen in the yeast Pichia pastoris forming highly immunogenic particles for cattle. Journal of Biotechnology, v. 33, p. 135-146, 1994.

RUIZ, P. M. G.; PASSOS, L. M. F.; RIBEIRO, M. F. B. Lack of infectivity of a Brazilian Anaplasma marginale isolate for Boophilus microplus ticks. Veterinary Parasitology, v. 128, p. 325-331, 2005.

SALES JUNIOR, P.; GUZMAN, F.; VARGAS, M.I.; SOSSAI, S.; PATARROYO, A. M.; LOMBANA, C.Z.G.; PATARROYO, J. Use of biodegradable PLGA microspheres as a slow release delivery system for the Boophilus microplus synthetic vaccine SBm7462. Veterinary Immunology and Immunopathology, v. 107, p. 281-290, 2005.

SANCHEZ, I.X.B. Estudo "in vitro" da apoptose induzida em linfócitos de camundongos (Balb/c) imunizados com o peptídeo sintético SBm7462. 2004. 79 p. Dissertação (Mestrado em Medicina Veterinária. Universidade Federal de Viçosa, Viçosa.

SANGSTER, N.C. Managing parasiticidae resistance. Veterinary Parasitology, v. 98, p. 89$109,2001$.

SANTOS, T. R. B.; GONZALES, J. C.; CHIES, J. M.; FARIAS, N. A. R. Transmissão transovariana de Babesia bigeminaI, (SMITH 7 KILBORNE, 1893) por partenóginas de Boophilus microplus (CANESTRINI, 1887). Revista Brasileira de Parasitologia Veterinária, v. 7, n. 1, p. 7-10, 1998.

SOSSAI, S.; PECONICK, A. P.; SALES-JUNIOR, P. A.; MARCELINO, F. C.; VARGAS, M. I.; NEVES, E. S.; PATARROYO, J. H. Polymorphism of the bm86 gene in South American strains of the cattle tick Boophilus microplus. Experimental and Applied Acarology, v. 37, p. 199214, 2005. 
SUTHERST, R. W. The vulnerability of animal and human health to parasites under global change. International Journal for Parasitology, v. 31, p. 933-948, 2001.

TAYLOR, M.A. Recent Developments in Ectoparasiticides. The Veterinary Journal, v. 161, p. 253-268, 2001.

WILLADSEN, P.; RIDING, G. A.; MCKENNA, R. V.; KEMP, D. H.; TELLAM, R. L.; NIELSEN, J. N.; LAHNSTEIN, J.; COBON, G. S.; GOUGH, J. M. Immunologic control of a parasitic arthropod. Identification of a protective antigen from $R$. (B.) microplus. Journal of immunology, v. 143, n. 4, 1346-1351, 1989.

WILLADSEN, P. Anti-tick vaccines. Parasitology, v. 129, p. 367-387, 2004.

WILLADSEN, P. Tick control: Thoughts on a research agenda. Veterinary Parasitology, v. 138, p. 161-168, 2006. 